\title{
Reality and Perception: Activity monitoring and data collection within a real-world smart home
}

\author{
Sarah Fallmann*, Ismini Psychoula*, Liming Chen*, Feng Chen*, Julie Doyle ${ }^{\dagger}$ and Darpan Triboan* \\ ${ }^{*}$ School of Computer Science and Informatics, De Montfort University, Leicester, UK \\ Email: \{sarah.fallmann, ismini.psychoula, liming.chen, fengchen\}@dmu.ac.uk, darpan.triboan@my365.dmu.ac.uk \\ ${ }^{\dagger}$ CASALA, Dundalk Institute of Technology, Co.Louth, Ireland \\ Email: julie.doyle@ casala.ie
}

\begin{abstract}
Smart home technologies have been developing rapidly in the last few years. However, there is still a lack of annotated rich datasets that can be used for different analysis purposes by researchers. The motivation for this study is driven by the need of self-management for chronic disease patients and the often neglected privacy aspects. The study describes the extension of an existing smart home environment at Great Northern Haven (GHN) with ambient and wearable devices. The discussed principles include the design of the experiment, data collection strategies and encountered challenges in regards to the sensors, connection problems and occupation with multiple inhabitants.
\end{abstract}

\section{INTRODUCTION}

The population in developed countries is ageing. For Europe this means, that the number of people above 65 years and older will increase significantly between 2015 and 2060 causing almost double the amount in this age range [8], [14]. Therefore, some severe issues need to be addressed including chronic diseases, which lead to the need of long term care. The impact of more elderly on society is not only based on costs, but is also linked to the lack of resources [8], [10]. This means in the future the number of caregivers will not be sufficient to meet the demands of elderly, trying to live independently or in care homes. The needs of elderly to stay at home independently as long as possible, are addressed in the field of Active and Assisted Living (AAL) which includes home automation systems and intelligent environments. In this context, information and communication technologies can help the interaction with people and use the information of sensors and actuators to detect where and what the people are doing at the moment, in order to avoid risky situations [8].

However, the advances in AAL rely increasingly on personal data. Such data are sensitive and therefore can lead to privacy risks such as privacy of behaviour and action, privacy of communication, privacy of data and image, privacy of location, and privacy of association [2], [7]. It is also difficult to find the right balance between individual privacy and availability of data. The main challenges are: (1) The well-being of elderly residents could be at risk if information is not accessible at the right time; (2) Private data could be shared between different systems putting the privacy of the resident at risk.

The overall aim of this research is to create an annotated dataset that combines data from ambient, wearable and binary sensors for multi-purpose analyses. The advantage of this data collection will be the enrichment of an existing environment with wearable and easy to apply ambient devices. This is an improvement towards a system which can be adjusted in private households providing independent living for individual purposes. The environment is designed to be highly adaptable and expendable at any time. The personalization will be based on the needs of the inhabitants including privacy aspects, chronic conditions and case of emergencies.

The study is structured in the following way. Section III discusses the related work in regards to the research topics addressed in this study and the data collection process. Section III describes the user scenarios, data collection process and activity monitoring. Section IV includes data prepossessing, cleaning and fusion of the dataset. In Section $\mathrm{V}$ challenges of the collection are outlined and discussed. Further work and conclusions are summarized in Section VI]

\section{RELATED WORK}

Ambient assisted living data collection methods are currently highly researched to allow elderly people, to live independently at home. There are a number of smart environments, some focus on a single occupied smart home while others involve multiple apartments with residents living there for years. Usually these environments monitor residents by using various sensors and creating algorithms to recognize their behaviours and extracting the information of daily living activities. However, most of the current research focuses on short activities that are mainly performed in laboratory environments. In these cases, the experiments are controlled and the activities are carried out based on the requirements of the research. But in a real world scenario it is difficult to predict the circumstances under which daily activities will occur [11]. There is also a lack of annotated reference datasets for researchers to apply and assess their models. The reason is that the monitoring of more complicated and even interleaved activities for a longer period of time in a real home environments is more difficult to achieve. That is because it requires continuous real-time data gathering, as well as storage of large amounts of information [1]. Data collection has been addressed by different research groups in laboratory settings as well as real world environments. There are smart home infrastructures 
which already exist to address the issue of having real world environmental settings [4], [6], [15]. Great Northern Haven (GNH) is such an infrastructure consisting of 16 smart homes equipped with ambient sensors and actuators. Older adults have permanently occupied fifteen of the apartments since 2010 while the remaining apartment is used for research and demonstration purposes [6]. These concepts mainly include motion/light sensors, door sensors and temperature sensors. To be adaptable to new rapidly growing technologies [12], infrastructures need to be highly flexible to different devices. This makes the approach applicable to every home and already existing environments. The infrastructure in [12] is designed for use cases of getting up, prepare meals using stove and kitchen sink. The proposed system in this study is designed for different research purposes and use cases including sleep quality, daily behaviour and privacy issues.

\section{Smart EnVironment Design And SEnsor DEPLOYMENT STRATEGIES}

This Section deals with the smart environment setup, monitored activities following an use case approach and sensor aspects including environmental and wearable devices.

\section{A. Smart Environment Setup}

The motivation for the environment design are (1) the selfmanagement for chronic diseases focusing on depressed and chronic kidney disease patients. This includes discussion of sleep efficiency calculation, self-management of fluid intake and enrichment of daily activity recording with a mobile Electroencephalography (EEG). The referred activities include go-to-bed routines and daily activities in the kitchen and living room. While the other aim (2) is to analyze the collected dataset from a privacy and security perspective, where the privacy risks will be evaluated based on the impact on individuals privacy. Threats will be identified by the use of smart home sensors, time, location, activity and health and wellness monitoring devices.

In this study, there are two basic sources of data. First, data from embedded sensors in the existing GNH apartment's infrastructure. Second, from the additional sources of data which include ambient and wearable sensors added in the environment, as shown in Figure 1 and 2. Data collection was performed for a continuous two weeks trial from two researchers within the extended real-world smart home.

\section{B. Activity Monitoring based on User Scenarios}

The study follows an use case approach where common daily behaviours were formulated in use cases and performed afterwards. Sensor data is collected for different user scenarios which are compatible for research topics of privacy, daily living and sleep quality.

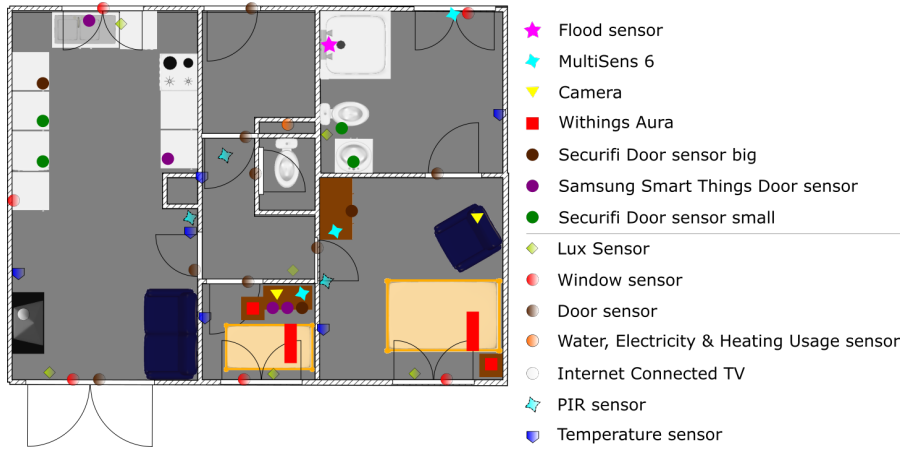

Fig. 1: Floor plan of DKIT smart environment with existing (semi-filled, framed) and extended (filled) smart sensors.

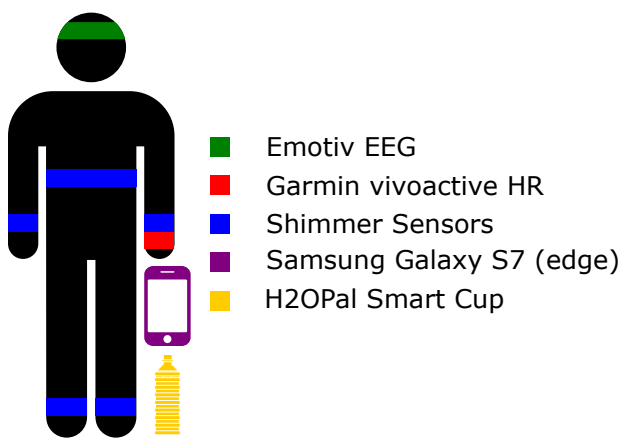

Fig. 2: Wearable devices used during the study and their respective location on the body.

1) Privacy: Privacy issues in the smart home environments appear to be one of the most important factors that can affect smart home technology adoption [3]. The use cases created for this experiment combine daily activities with different privacy invasive technology elements. In addition to the behaviour pattern analysis of these elements, they will also be investigated from a privacy point of view. The collected data will be analyzed based on the sensor type, the location, time, activity while metric algorithms will be developed to measure the sensitivity of the information they reveal and the potential risk depending on who could have access to it.

2) Sleep Quality: Sleep quality is an important aspect of wellbeing, especially for elderly [5]. This aspect is one focus of the study which is analysed including nighttime (Table I) and daytime (Table III) sleeping patterns. Important features will be extracted representing sleep quality to further apply them in a weight function to represent night's rest. These use cases are designed for relevant conditions including healthy elderly without problems, a depressed patient suffering from insomnia and a chronic kidney disease patient (compare Table II. Usually it is advised to keep nap duration during the day under 30 minutes [13], as it effects the sleep quality during the night. This topic is discussed controversially in the literature [9]. Therefore this open issue needs further detailed research, which is incorporated in the study by the use case described in Table III. Data collected from the different user scenarios will 
TABLE I: Use case "Sleep Quality assessment of healthy elderly and chronic disease patients

\begin{tabular}{|c|c|}
\hline Description & $\begin{array}{l}\text { The use case describes sleep quality measurements } \\
\text { within a smart environment including environmental and } \\
\text { wearable sensors for patients in different conditions. }\end{array}$ \\
\hline Conditions: & $\begin{array}{l}\text { Patient [healthy(HEA), depressed(DEP) suffering from } \\
\text { insomnia and suffering from chronic kidney dis- } \\
\text { ease(CKD)], Smart Home } \\
\text { Sleeping behaviour: } \\
\text { HEA normal } \\
\text { DEP problem related to lack of sleep, difficulties } \\
\text { falling asleep(onset), staying asleep(maintenance) } \\
\text { or waking up too early. } \\
\text { CKD problem related to waking up during the night, } \\
\text { sleep disturbances, need to use the toilet caused } \\
\text { by medication and fluid consumption. }\end{array}$ \\
\hline $\begin{array}{l}\text { Action } \\
\text { sequence: }\end{array}$ & $\begin{array}{ll}\text { 1) Adjust required sensors for sleep. } \\
\text { HEA,DEP } & \text { Withings Aura, Smartphone, camera } \\
\text { HEA,CKD } & \text { Shimmer sensors, Smart-Watch, } \\
& \text { Withings Aura, Smartphone } \\
\text { 2) HEA } & \text { The person falls asleep . } \\
\text { DEP,CKD } & \begin{array}{l}\text { The person has difficulties to fall } \\
\text { asleep. }\end{array} \\
\text { 3) DEP } & \begin{array}{l}\text { The person wakes up during the } \\
\text { night and has difficulties falling } \\
\text { asleep again. } \\
\text { The person wakes up during the }\end{array} \\
& \text { night to go to the bathroom. }\end{array}$ \\
\hline
\end{tabular}

TABLE II: Use case "Napping during the day"

\begin{tabular}{l|l}
\hline Description & The use case is related to napping during the day. \\
\hline $\begin{array}{l}\text { Actors } \\
\text { Conditions: }\end{array}$ & $\begin{array}{l}\text { Elderly, Smart Home } \\
\text { Ection }\end{array}$ \\
$\begin{array}{l}\text { sequence: } \\
\text { 1) Elderly adjusts sensors including Smart Watch, } \\
\text { Shimmer Sensors, Withings Aura, Smartphone } \\
\text { The person naps inside the bedroom. }\end{array}$
\end{tabular}

TABLE III: Use case "Fluid Consumption"

\begin{tabular}{l|l}
\hline Description & $\begin{array}{l}\text { The use case illustrated the fluid consumption for an } \\
\text { elderly person using a Smart Cup. }\end{array}$ \\
\hline $\begin{array}{l}\text { Actors } \\
\text { Condition: } \\
\text { Action } \\
\text { sequence: }\end{array}$ & $\begin{array}{l}\text { Elderly person, Smart Home } \\
\text { Person is thirsty. }\end{array}$ \\
& $\begin{array}{l}\text { 1) The person feels thirsty. } \\
\text { 2) The person goes to kitchen to fill the SmartCup } \\
\text { with a drink. }\end{array}$ \\
& $\begin{array}{l}\text { 3) The person drinks. } \\
\text { 4) Sensors in the SmartCup measures the quantity the } \\
\text { person drunk. }\end{array}$ \\
\hline
\end{tabular}

TABLE IV: Use case "Physical Activity of an Eldery Wearable Monitoring"

\begin{tabular}{|c|c|}
\hline Description & $\begin{array}{l}\text { The use case is related to the physical activity of an } \\
\text { elderly during the day. }\end{array}$ \\
\hline $\begin{array}{l}\text { Actors } \\
\text { Conditions: } \\
\text { Action } \\
\text { sequence: }\end{array}$ & $\begin{array}{l}\text { Elderly, Smart Home } \\
\text { Elderly is inactive. } \\
\text { 1) The person adjusts wearable device (Smart Watch) } \\
\text { on the body day and night. } \\
\text { 2) The environment analyses the changes of presence } \\
\text { inside the house as well as the Smart Watch data } \\
\text { in and outside the house. }\end{array}$ \\
\hline
\end{tabular}

TABLE V: Use case "Hygiene - bed time and morning routine"

\begin{tabular}{|c|c|}
\hline Description & $\begin{array}{l}\text { The use case describes hygiene for an elderly person } \\
\text { including environmental and wearable sensors during the } \\
\text { bed time and morning routine. }\end{array}$ \\
\hline $\begin{array}{l}\text { Actors } \\
\text { Conditions: } \\
\text { Action } \\
\text { sequence: }\end{array}$ & $\begin{array}{l}\text { Elderly person, Smart Home } \\
\text { Elderly wants to go to bed or get up. } \\
\text { 1) The person adjusts required sensors on the body } \\
\text { during the bathroom routine. (Smart Watch, Shim- } \\
\text { mer Sensors) } \\
\text { 2) The person enters the bathroom } \\
\text { 3) The environment measures the presence in the } \\
\text { bathroom, use of the tap, use of toilet, use of } \\
\text { shower and the accelerometer from Shimmer sen- } \\
\text { sors. } \\
\text { 4) The person starts the bathroom routine including } \\
\text { comb hair, wash face and hands, brush teeth, use } \\
\text { the toilet and shower if necessary }\end{array}$ \\
\hline
\end{tabular}

TABLE VI: Use case "Daily Activities"

\begin{tabular}{l|l}
\hline Description & $\begin{array}{l}\text { The use case describes elderly person daily routine } \\
\text { including prepare meal/tea, eat, drink, watch television. }\end{array}$ \\
\hline $\begin{array}{l}\text { Actors } \\
\text { Conditions: } \\
\text { Action } \\
\text { sequence: }\end{array}$ & $\begin{array}{l}\text { Elderly person, Smart Home } \\
\text { Elderly person performs daily activities. }\end{array}$ \\
1) The person adjusts the EEG Emotiv to measure \\
daily activities. \\
2) Activities are performed: prepare meal, prepare \\
tea, drink, eat, watch television, work \\
3) Emotiv device collects EEG data during the ac- \\
tivities and the environment helps to clarify the \\
activities performed. \\
4) The person takes off the Emotiv.
\end{tabular}

TABLE VII: Use case "Electricity, Water and Heating Usage Monitoring"

\begin{tabular}{l|l}
\hline Description & $\begin{array}{l}\text { The use case describes the monitoring of data through } \\
\text { consumption meters. }\end{array}$ \\
\hline $\begin{array}{l}\text { Actors } \\
\text { Conditions: }\end{array}$ & $\begin{array}{l}\text { Elderly person, Smart Home } \\
\text { The smart house is monitoring the consumption in real } \\
\text { time }\end{array}$ \\
$\begin{array}{l}\text { Action } \\
\text { sequence: }\end{array}$ & $\begin{array}{l}\text { 1) The smart home system aggregates information } \\
\text { from many different devices } \\
\text { The user can see their consumption statistics } \\
\text { This information could potentially reveal sensitive } \\
\text { information about the resident }\end{array}$ \\
\hline
\end{tabular}


be analyzed to extract further information for sleep efficiency.

3) Daily Behaviour: This Section refers to daily behaviour of elderly, divided into sleep hygiene and daily behaviour aspects.

i Sleep hygiene is used as treatment for insomnia [13], [16] which includes

light exposure measured via light sensors inside the house and the time spent outside

exercises amount measured with environmental sensors and Smart Watch, see Table IV ing caffeine consumption annotation, compare Table III

environment checking of noise level, humidity and temperature inside the room

nap duration, refer to Table II

routine for bedtime is checked by environmental and wearable sensors, see Table V

ii General performed activities during the day in the

bathroom use toilet, shower, wash face, brush teeth, comb hair

kitchen prepare food, eat, drink, wash-up

bedroom sleep, nap, putting blinds up/down, making bed, dress

livingroom watching TV, working, reading

The daytime activities were not only measured by environmental sensors but also extended by EEG measurements described in the use case in TableVI. In Table VII the use case to monitor electricity, water and heating usage is illustrated.

\section{Sensor Deployment and Data Collection}

The existing smart home at GNH consists of environmental wired sensors including window, door, consumption usage, PIR, lux and temperature sensors (refer to Table VIII). This

TABLE VIII: Existing smart environment setting from DKIT, Abbreviation $\mathrm{N}$ indicates the number of sensors

\begin{tabular}{l|lll}
\hline Sensor type & N & Location & Detection \\
\hline Doors sensors & 7 & doors & open/close \\
Window sensors & 5 & windows & open/close \\
Electricity, water \& heating usage & 1 & boiler & usage \\
PIR sensors & 3 & ceiling & movement \\
Lux sensors & 6 & ceiling & light \\
Temperature sensors & 6 & walls & temperature \\
\hline
\end{tabular}

smart home was extended by wearable and environmental devices like door, movement and flood sensors as well as Shimmer sensors and Withings Aura, listed in Table IX The location of existing and extended sensors within the flat is shown in Figure 1. In Figure 2 the wearable devices are depicted those applied on the body like Shimmer sensors, Emotiv EEG and Smart Watches as well as Smartphones and Smart Cups.
TABLE IX: Extended wearable and environmental sensors for the smart environments at GNH. The abbreviations R1, R2 stand for bedrooms of the residents, $\mathrm{K}$ for kitchen, $\mathrm{B}$ for bathroom and $\mathrm{N}$ for the number of sensors.

\begin{tabular}{|c|c|c|c|}
\hline Sensor type & $\mathbf{N}$ & Location & Collected for \\
\hline Shimmer Sensors & 5 & extremities, chest & daily activities, sleep \\
\hline Camera & 2 & bedroom(R1,R2) & annotation \\
\hline Smart Watch & 1 & wrist(R1) & sleep, active \\
\hline Withings Aura & 2 & bedroom(R1,R2) & sleep \\
\hline Samsung Galaxy & 2 & bed(R1,R2) & sleep \\
\hline IPhone & 2 & smart home & annotation \\
\hline H2OPal Smart Cup & 2 & smart home & fluid intake \\
\hline EEG Emotiv & 1 & head(R1) & daily activities \\
\hline Securifi Door & 3 & $\operatorname{cupboard}(\mathrm{K})$, & open/close \\
\hline Sensors big & & wardrobe(R1,R2) & \\
\hline Securifi Door & 4 & fridge $(\mathrm{K})$ & open/close \\
\hline Sensors small & & $\begin{array}{l}\text { cupboard(K), } \\
\text { toilet(B), tab(B) }\end{array}$ & \\
\hline Samsung Smart & 4 & $\operatorname{tab}(\mathrm{K})$ & open/close \\
\hline Things Door & & $\operatorname{cupboard}(\mathrm{K})$, & \\
\hline Sensors & & wardrobe (R1) & \\
\hline MultiSensor6 & 3 & $\begin{array}{l}\text { bedrooms(R1,R2), } \\
\text { bathroom }\end{array}$ & $\begin{array}{l}\text { movement, } \\
\text { temperature, light, } \\
\text { humidity, vibration }\end{array}$ \\
\hline Flood Sensor & 1 & bathroom & water \\
\hline
\end{tabular}

Two different settings were designed for sleep quality assessment. In one setup Withings Aura and a Smartphone under the pillow is collecting data over the entire night. The second setting extends the first setup with a Smart Watch and Shimmer sensors applied to the extremities and the chest. The second setup is gathering data during the night and 3045 minutes long naps during the day. This Shimmer sensor collects accelerometer, gyroscope, magnetometer, pressure and temperature data. The Garmin vivoactive HR Smart Watch gathers data about sleep stages, heart rate and movement, whereas Withings Aura uses an air pillow under the mattress to calculate sleep stages, heart rate, temperature, luminosity and noise. The integrated Smartphone sensors collect data in 0.1 to 1 second updating rate. Fluid consumption is measured with H2O Pal Smart Cup, gathering milliliter amount drunk during the day. Emotiv EEG is collecting data of daytime activities like preparing food, eating, making tea, drinking, watching television or working on a laptop. Additionally, binary sensors in the flat record different daily activities continuously (compare Section III-B3).

\section{DAta Processing}

This Section describes the processing of the dataset which is currently in progress.

\section{A. Data Pre-Processing and Cleaning}

The data pre-processing of the collected dataset will include data cleaning to remove erroneous and unwanted values, data interpolation to deal with potential missing values and data transformation to put the data into the correct formats. For example, the values for temperature and date are in different 
formats based on the type of sensor.

As the collected data are from different types of sensors in a real environment there is the possibility that noisy and redundant information is generated, for instance by discharged batteries, failures in sensor readings and losses in wireless transmission, as well as unforeseen events that occur in real homes. To perform data cleaning, several filters will be used which depend on the characteristics of the sensor and the amount of people that were present. For example Bayes and particle filters will be used to clean data from binary sensors in scenarios with multiple residents present. A median filter will be used to remove noise and avoid abnormal measurements from the presence sensors. The noisy data collected from EEG and Shimmer devices will be reduced by signal processing. For the accelerometer a low pass filter will be used to remove the gravity component from the values and high pass filters to reveal the real acceleration.

\section{B. Data Processing}

Data from different devices is combined and related to activities within the smart environment using timestamps from sensors and timestamps from annotation from Smartphone and camera. The Smartphone contains a customised application that allows the annotation of daily activities and their duration. Camera data are used to get a ground truth for sleep movement and annotating activities during EEG recordings. Smartphone annotation is mainly used for daily activities mainly in the bathroom, kitchen and bedroom (compare Section III-B3.

Before analyzing the collected data a risk model will be developed that will be able to estimate privacy risks. The model will include equations that take into account various data inputs and parameters, and then calculate the likelihood of potential privacy problems. This model will allow the identification of privacy risks for the residents of smart homes that could arise from the processing of personal information the collected data contain.

Feature selection will be performed for Shimmer senors and EEG recordings to extract relevant factors to be included in activity recognition. For Shimmer data this activities include daily activities as well as respiration, movement and sleep position. The frequency of sensor collection is deviate. For instance, Shimmer sensors on the extremities are collected with a frequency of $50 \mathrm{~Hz}$ and on the chest with $520 \mathrm{~Hz}$ and Smartphone sensor gathering has a frequency of $1-10 \mathrm{~Hz}$. Therefore they will be processed independently and the outcomes will be combined. The Shimmer chest sensor is used for respiration detection with the gathered frequency. Whereas for position during the sleep the lowest frequency will be set to all of the Shimmer devices including extremities and chest. Withings Aura, Shimmer, Smart Watch, Smartphone device recordings and environmental devices will be included in the sleep quality calculation. This calculation is based on a weight function using temperature, humidity, movement, heart rate, sleep stages, noise level, light level, bed exists and physical activity during the day. Physical exercise can effect the sleep positively [9]. Therefore, the Smart Watch is used to extract an activity level outside the home which will use steps and heart rate during the day. Inside the home the activity level is calculated based on the movement sensor activations. Fluid intake will be analyzed using the Smart Cup data. Activity recognition is applied on EEG recording and environmental sensors. The change in behaviour after a good and bed night's rest will be represented correlating outputs from the single data processing steps.

\section{Discussion AND Challenges}

The focus of this Section are the challenges which had to be faced during the study and how they are addressed during the processing of data. This challenges include the sensor setup, data collection, extraction and processing.

1) Hardware pre-setup: An ideal setting was established which included binary and wearable sensors to be integrated in the existing environment. At the start of the experiment some difficulties were encountered in applying and pairing some sensors in the home which had to be excluded in further investigations. This included movement and door sensors. Some sensors were not easy to install as their purpose was changed. Door sensors were used as sink tap sensors gathering data about temperature and detecting water flow. This was difficult to apply in the bathroom and kitchen setting.

2) Connection difficulties: Failures of Bluetooth connection and synchronization between wearable devices and Smartphones had to be faced during the real world environmental study. Other difficulties are possible Internet connection problems, as some devices need this for their setup. The SmartCam and Aura Withings were connected to the Internet to interact with the cloud systems of the providers. This camera setup allows not only the collection of ground truth, but also creates a very privacy invasive scenario by uploading private video recording to a company's cloud service.

3) Malfunction: Device software challenges were another issue, sometimes specific devices stopped recording data prematurely after a short duration or failed to connect correctly. These difficulties lead to a lack of data during the experiment, which will be met by an algorithmic methodology that can handle incomplete and noisy datasets.

4) Data extraction: After activating the sensors the data collection started, which was interrupted by some problems with data transition and raw data extraction from third party device providers. This means that some providers, do not give access to the whole raw dataset from their devices. Other devices have a complicated structure of output format which has to be studied in detail to understand the overall output.

5) Data processing: The data processing step faces some difficulties in correlating between timestamps and frequency differences. The annotated information needs to be correlated 
carefully, as some activities started earlier than the corresponding collection of data. The formats of the different extracted datasets from providers have to be adjusted to be comparable and able to integrate.

6) Occupation: The occupation of the smart home in regards to the number of persons varied during the study. The main challenge during data processing is the extraction of behaviour patterns based on the inhabitants living in the smart home. Also, visits to the smart environment from guests and external researchers took place, which have to be taken into account during the data processing step. This situation affects data collection from the environmental devices.

\section{CONClusion AND Future Work}

In this study a dataset is collected within a real world environment, that enables to conduct further research in context of sleep, daily behaviour and privacy aspects. An existing smart home at GNH equipped with wired sensors was extended by a combination of wearable and environmental devices. The gathering was continuously performed over a period of two weeks with two permanent inhabitants. Due to the setting including various devices, multiple use cases can be addressed like hygiene, fluid consumption, wearable monitoring, sleep, daily activities and energy consumption. The challenges during the performance of the study have been the sensor setup, occupation, data extraction and processing.

Collected data is going to be applied to algorithms extracting features for sleep quality and behaviour analysis based on environmental and wearable devices. First a sleep efficiency calculation will be performed using parameters extracted from wearable and ambient devices. Further both aspects will be linked to each other representing the changes on behaviour after a high and low rated night's rest. The idea is to extract factors which could be dealt with further in sleep efficiency calculations or be replaced by non-contact devices.

Further on, a framework will be developed to identify the privacy risks based on who will access the data, in which context data will be used and what the residents benefits or risks will be from reveling this data. In order to estimate the privacy impact, models will be developed that use identified risks associated to the collected data. The probability that these data become problematic for a resident whose personal information is processed and possibly shared by the system will be calculated. The sensitivity of the information will be analyzed based on how sensitive data is perceived to be depending on the circumstances and varying personal preferences.

\section{ACKNOWLEDGMENT}

This work has been funded by the European Union Horizon2020 MSCA ITN ACROSSING project (GA no. 616757). The authors would like to thank the members of the project consortium for their valuable inputs. We would also like to show our gratitude to Dundalk Institute of Technology for hosting us in the Great Northern Haven, Netwell-CASALA research smart home and providing their help and knowledge.

\section{REFERENCES}

[1] Kevin Bouchard and Sylvain Giroux. Smart homes and the challenges of data. In Proceedings of the 8th ACM International Conference on PErvasive Technologies Related to Assistive Environments, page 66. ACM, 2015.

[2] Patrice Caire, Assaad Moawad, Vasilis Efthymiou, Antonis Bikakis, and Yves Le Traon. Privacy challenges in ambient intelligence systems. Journal of Ambient Intelligence and Smart Environments, 8(6):619-644, 2016.

[3] Jane Chung, George Demiris, and Hilaire J Thompson. Ethical considerations regarding the use of smart home technologies for older adults: an integrative review. Annual review of nursing research, 34(1):155-181, 2016.

[4] Aaron S. Crandall, Narayanan C. Krishnan, Brian L. Thomas, and Diane J. Cook. Casas: A smart home in a box. Computer, 46:62-69, 2013.

[5] M. Jamal Deen. Information and communications technologies for elderly ubiquitous healthcare in a smart home. Personal and Ubiquitous Computing, 19(3):573-599, 2015.

[6] Julie Doyle, Andrea Kealy, John Loane, Lorcan Walsh, Brian O'Mullane, Carl Flynn, Andrew Macfarlane, Brennon Bortz, R Benjamin Knapp, and Rodd Bond. An integrated home-based self-management system to support the wellbeing of older adults. Journal of ambient intelligence and smart environments, 6(4):359-383, 2014.

[7] Rachel L Finn, David Wright, and Michael Friedewald. Seven types of privacy. In European data protection: coming of age, pages 3-32. Springer, 2013.

[8] Francisco Florez-Revuelta and Alexandros Andre Chaaraoui(Eds.). Active and Assisted Living: Technologies and Applications. IET, 2016.

[9] Leah A. Irish, Christopher E. Kline, Heather E. Gunn, Daniel J. Buysse, and Martica $\mathrm{H}$. Hall. The role of sleep hygiene in promoting public health: A review of empirical evidence. Sleep Medicine Reviews, 22:23 $-36,2015$

[10] Nelda McCall. Who Will Pay for Long Term Care? Insights from the Partnership Programs. Health Administration Press, 2001.

[11] Qin Ni, Ana Belén García Hernando, and Iván Pau de la Cruz. The elderlys independent living in smart homes: A characterization of activities and sensing infrastructure survey to facilitate services development. Sensors, 15(5):11312-11362, 2015.

[12] Thomas Reichherzer, Steven Satterfield, Joseph Belitsos, Janusz Chudzynski, and Lamar Watson. An Agent-Based Architecture for Sensor Data Collection and Reasoning in Smart Home Environments for Independent Living, pages 15-20. Springer International Publishing, Cham, 2016.

[13] Edward J Stepanski and James K Wyatt. Use of sleep hygiene in the treatment of insomnia. Sleep Medicine Reviews, 7(3):215 - 225, 2003.

[14] European Union. The 2015 Ageing Report. Underlying Assumptions and Projection Methodologies Technologies and Applications for Active and Assisted Living. Current situation, 2014.

[15] Tim van Kasteren, Athanasios Noulas, Gwenn Englebienne, and Ben Kröse. Accurate activity recognition in a home setting. In Proceedings of the 10th International Conference on Ubiquitous Computing, UbiComp '08, pages 1-9, New York, NY, USA, 2008. ACM.

[16] Annemieke van Straten, Tanja van der Zweerde, Annet Kleiboer, Pim Cuijpers, Charles M. Morin, and Jaap Lancee. Cognitive and behavioral therapies in the treatment of insomnia: A meta-analysis. Sleep Medicine Reviews, pages -, 2017. 